\title{
A Progressive Gradient Moment Nulling Design Technique
}

\author{
JAmes G. Pipe AND Thomas L. Chenevert \\ University of Michigan Medical Center, 1500 East Medical Center Drive, Ann Arbor, Michigan 48109
}

Received February 9, 1990; revised May 16, 1990

\begin{abstract}
A method is presented for designing motion-compensated gradients in a progressive manner. The method is easily applicable to many types of waveforms, and can compensate for any order of motion. It can be implemented graphically or numerically. Underlying theory and examples of its application are provided. (c) 1991 Academic Press, Inc.
\end{abstract}

\section{INTRODUCTION}

Motion of spins during the application of gradients gives rise to incremental phase drifts relative to static spins ( 1 ). Gradient moment nulling (GMN) techniques have been described in the literature (2-4) to control motion-related phase shifts through proper gradient waveform design. At least three design methods have been proposed (5-7). Keller suggests the use of the binomial expansion coefficients for gradient amplitudes; this is easily applicable, but limits the possible waveforms. Xiang has presented a matrix equation for GMN which is a more powerful technique, but is also computationally involved and limits possible waveforms to a linear combination of a predefined set of basic gradient waveforms. Chang uses electrical multipoles as an aid in analyzing moments of gradient pulses. In this discussion a simple analysis of the problem of GMN is described along with a waveform design method that is easily applicable and very flexible, allowing a wide range of waveforms to be constructed. The method is an iterative one, which allows one to take a gradient compensated for some order of motion (e.g., velocity) and quickly form many waveforms which are compensated for the next order of motion (e.g., acceleration). Therefore one can start with an arbitrary "static position-compensated waveform, and easily build many waveforms which are compensated for velocity, acceleration, jerk, etc.

The flexibility and ease of application of this method is important, since the pulse sequence designer must not only design for motion compensation, but must also consider imposed gradient functions (e.g., slice selection, readout), avoid undesired affects such as unwanted echoes, and design for performance within system limitations. With this method, one can create a host of pulses to choose from to suit individual needs. A brief proof of the mathematics behind this method is provided, followed by some examples of its use.

\section{THEORY}

The relative phase $\phi$ of a spin is given by

$$
\phi=\gamma \int G(t) x(t) d t,
$$


where $x(t)$ is the position of the spin and $G(t)$ is the gradient waveform. The position of the spin can be expressed as a polynomial in $t$, i.e.,

$$
x(t)=\sum k_{i} t^{i}
$$

For $i=0, x(t)=k_{0}$, which gives a static position. Similarly, $x(t)=k_{1} t$ is the constant velocity term, $x(t)=k_{2} t^{2}$ gives constant acceleration, and so on. A gradient waveform $G_{\mathrm{c}}$ which is compensated for all motions up to order $n$, but uncompensated for order $n+1$ motion, has the properties

$$
\gamma \int G_{\mathrm{c}}(t) k_{i} t^{i} d t=0, \quad 0 \leqslant i \leqslant n \quad\left(\text { for any } k_{i}\right. \text { ) }
$$

and

$$
\gamma \int G_{\mathrm{c}}(t) k_{n+1} t^{n+1} d t=\partial
$$

where $\partial$ is the phase shift due to the uncompensated order of motion. It will be important to verify that the phase $\partial$ in [4] is independent of the time that $G_{\mathrm{c}}(t)$ is applied. To do this, consider [4] with the gradient shifted by a time $\tau$. This gives a phase of

$$
\phi=\gamma \int G_{\mathrm{c}}(t-\tau) k_{n+1} t^{n+1} d t
$$

Let $u=t-\tau$, which gives $d u=d t$. Since the integral limits are over all $t,[5]$ becomes

$$
\begin{aligned}
\phi & =\gamma \int G_{c}(u) k_{n+1}(u+\tau)^{n+1} d u \\
& =\gamma \int G_{c}(u) k_{n+1}\left(u^{n+1}+c_{n} U^{n} \tau+\cdots+c_{1} u \tau^{n}+(\tau)^{n+1}\right) d u \\
& =\gamma \int G_{\mathrm{c}}(u) k_{n+1} u^{n+1} d u+\gamma \int G_{\mathrm{c}}(u) k_{n+1}\left(c_{n} U^{n} \tau+\cdots+c_{1} U \tau^{n}+(\tau)^{n+1}\right) d u \\
= & =\partial+0=\partial .
\end{aligned}
$$

Note that the second integral equals zero since all the terms of $x(t)$ (of order $n$ and less) are motion compensated, as shown in condition [3]. Thus the phase error after a shift of $\tau$ is the same as the phase error $\partial$ in [4]. Put another way, the phase error is independent of the time $\tau$ the gradient is applied (for motion which is one order above the compensated motions). If one applies $G_{\mathrm{c}}(t)$ at time $a$, and in the same sequence adds the gradient $-G_{\mathrm{c}}(t)$ at time $b$, the total phase error (due to the $k_{n+1} t^{n+1}$ term of $x(t))$ is

$$
\begin{aligned}
\int G_{\mathrm{c}}(t-a) k_{n+1} t^{n+1} d t & +\int-G_{\mathrm{c}}(t-b) k^{n+1} t^{n+1} d t \\
& =\int G_{\mathrm{c}}(t) k_{n+1} t^{n+1} d t+\int-G_{\mathrm{c}}(t) k_{n+1} t^{n+1} d t=\partial-\partial=0 .
\end{aligned}
$$

This is an important result since [7] shows that a new "composite" gradient $G_{\mathrm{c}}^{\prime}(t)$ given by 


$$
G_{\mathrm{c}}^{\prime}(t)=G_{\mathrm{c}}(t-a)-G_{\mathrm{c}}(t-b)
$$

is now compensated for the $t^{n+1}$ term of $x(t)$. The entire process can be repeated; that is, $G_{\mathrm{c}}^{\prime}(t)$ can be used to compensate for the $t^{n+2}$ term of $x(t)$ by making a gradient

$$
G_{\mathrm{c}}^{\prime \prime}(t)=G_{\mathrm{c}}^{\prime}(t-c)-G_{\mathrm{c}}^{\prime}(t-d)
$$

This process can be iterated until the desired amount of motion compensation is obtained. Note that in this work, the effective sign inversion of gradient pulses (along with the relative phase $\phi$ ) due to $180^{\circ}$ pulses has been ignored for simplicity.

\section{METHODS}

\section{A. Graphical}

The method being described can be implemented using simple sketches of waveforms, without any calculation needed. As a simple example, consider the static position-compensated waveform shown in Fig. 1a, and its negative in Fig. 1b. These may be added together with arbitrary time shifts between them to give different velocity-

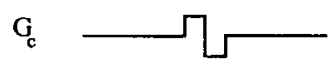

(a)

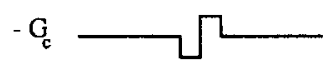

(b)

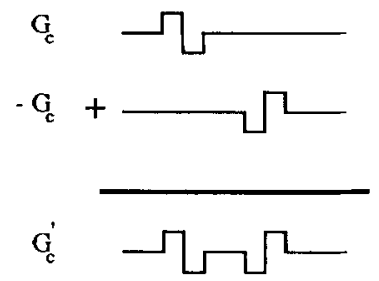

(c)

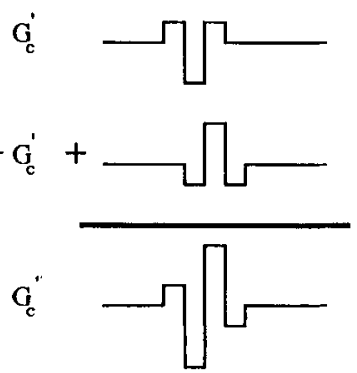

(f)

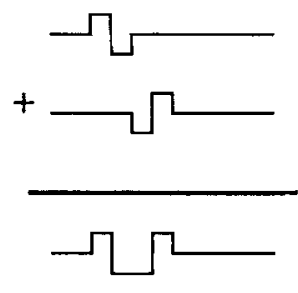

(d)
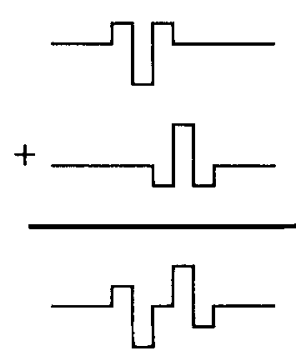

(g)

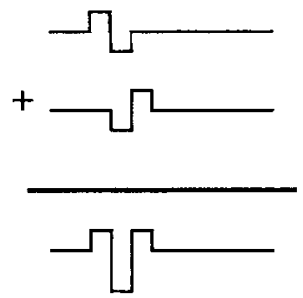

(e)
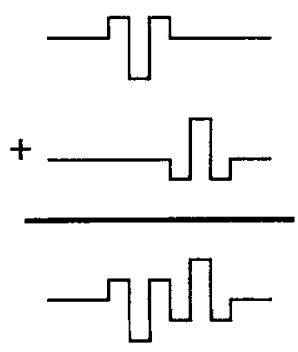

(h)

FIG. 1. A simple "static position"-compensated waveform (a) and its negative (b) are added together with different time shifts between them to make velocity-compensated waveforms $(c, d, e)$. The velocitycompensated waveform (e) and its negative are then added with different time shifts to make accelerationcompensated waveforms ( $f, g, h)$. 
compensated waveforms, as seen in Fig. 1 (c, d, e). One may also take a velocitycompensated waveform (for example, the waveform in Fig. 1e) and add it to its negative in a number of ways to get acceleration-compensated waveforms. Three examples of this are shown in Fig. 1 ( $f, g, h)$.

One need not begin with symmetric square pulses. Taking an uneven rectangular static position-compensated waveform can yield an entirely different velocity compensated waveform, as shown in Fig. 2. Alternatively, one could start with a more exotic waveform, such as a hyperbolic gradient, which may be desired for a readout gradient (8). Figure 3 shows velocity- and acceleration-compensated waveforms for this case. One can see that by varying both the starting waveform and the shifts used to add waveforms, a large variety of compensated waveforms can be designed.

\section{B. Numerical}

An alternative to the graphical implementation of this method with square pulses is to write them numerically. One can again start with the simple waveform in Fig. $1 \mathrm{a}$, and designate it as $1 \underline{1}$ ( static position compensated). Its negative is 11 . To get a velocity-compensated waveform, one needs only to shift them in time and add them up; i.e.,

$$
\begin{array}{llll}
1 \underline{1} & 1 \underline{1} & 1 \underline{1} & 1 \underline{1} \\
\frac{1}{00} & \frac{1}{12} 1 & \frac{1}{1 \underline{11} 1} & \frac{\underline{11}}{1 \underline{10} \underline{1} 1 .}
\end{array}
$$
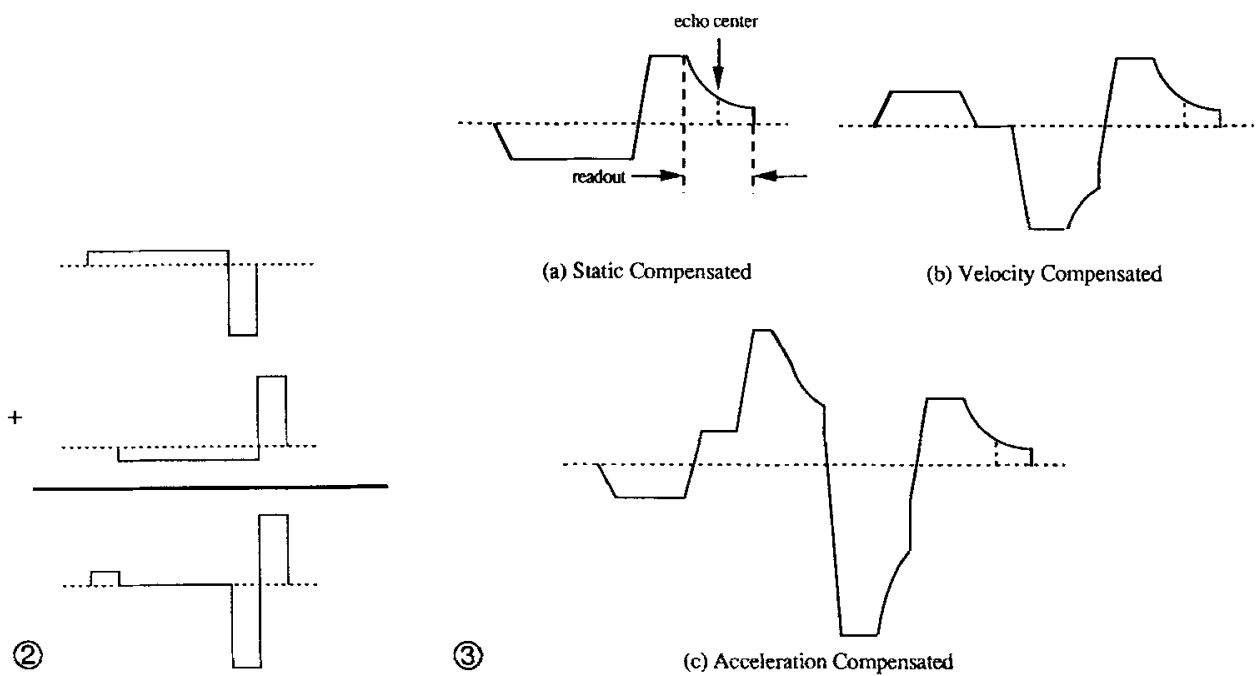

(a) Static Compensated

(b) Velocity Compensated

(3)

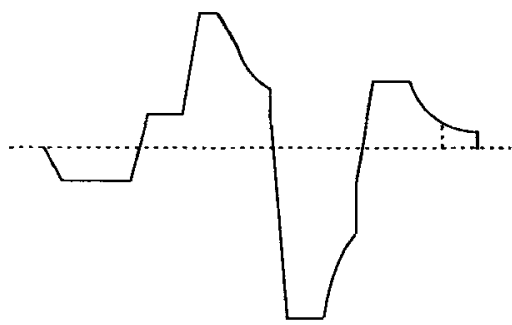

(c) Acceleration Compensated

FIG. 2. The formation of a velocity-compensated waveform by the addition of an assymetric staticcompensated waveform to its negative.

FIG. 3. Three possible waveforms for a gradient with the constraint of a hyperbolic readout period; (a) static compensated, (b) velocity compensated, (c) acceleration compensated. Dashed vertical lines indicate echo center. 
All of the above waveforms are velocity compensated. Take one (e.g., 121) and make an acceleration-compensated waveform as shown below.

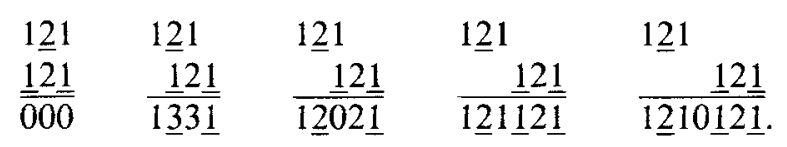

All of the above waveforms are acceleration compensated. The numerical method for the waveform in Fig. 2 is

$$
\begin{aligned}
& 11111 \underline{5} \\
& \underline{111115} \\
& \hline 10000 \underline{6} 5 .
\end{aligned}
$$

The numerical method cannot be applied to more complex waveforms, such as the one in Fig. 3. It requires waveforms made of one geometric shape whose magnitude can be expressed as a single number ( such as a square pulse or a sine bell), and only allows shifts in time of discrete amounts. When suitable, however, this method is very convenient for a fast look at possible waveforms.

\section{DISCUSSION}

The method described here does not generate all possible waveforms. It is presented as an easy tool for generating motion-compensated waveforms for different applications with little effort. For illustrative purposes, imagine that the velocity-compensated waveforms in Fig. 1 (c, d, e) are to be used for readout gradients (with the echo center occurring at the very end of the waveform). The waveform in Fig. 1c may be desired when a slice selective $180^{\circ}$ pulse is needed in the middle of the waveform, requiring a period of zero readout gradient strength. The waveform in Fig. 1e may be desired when time is critical, such as in a short TE gradient-recalled echo sequence. If one lacks strong enough gradients to achieve amplitudes three times the readout gradient amplitude, or if the slew rate of the gradients cannot allow this waveform, the waveform in Fig. 1d may be the preferred waveform.

Although the principles involved in this method are simple, it can be a powerful tool for those who design pulse sequences. It can be applied to most any sequence, does not even require a hand calculator for computation, and can produce many possible waveforms which are guaranteed to be motion compensated up to the desired order of motion.

\section{REFERENCES}

1. C. L. Schultz, R. J. Alfidi, A. D. Nelson, S. Y. Kopiwoda, And M. E. Clampitt, Radiology 152, 117 (1984).

2. P. M. Pattany, J. J. Phillips, L. C. Chiu et al. J. Comput. Assist. Tomogr. 11, 369 (1987).

3. A. D. ELSTER, $A J N R$ 9,671 (1988).

4. J. D. Lipcamon, L. C. Chiu, J. J. Phillips, and P. M. Pattany, Radiol. Tech 59(5), 415 (1988).

5. P. J. Keller AND F. W. Wehrli, J. Magn. Reson. 78, 145 (1988).

6. Q. S. XIANG AND O. NAlcioglu, in "Proceedings, 5th Annual Meeting of the Society of Magnetic Resonance in Medicine," p. 100, 1986.

7. R. Chang, A. DWyer, Radiology 165, 417 (1987).

8. H. N. Yeung, S. D. Swanson, And T. L. Chenevert, in "Proceedings, 8th Annual Meeting of the Society of Magnetic Resonance in Medicine," p. 8781989. 